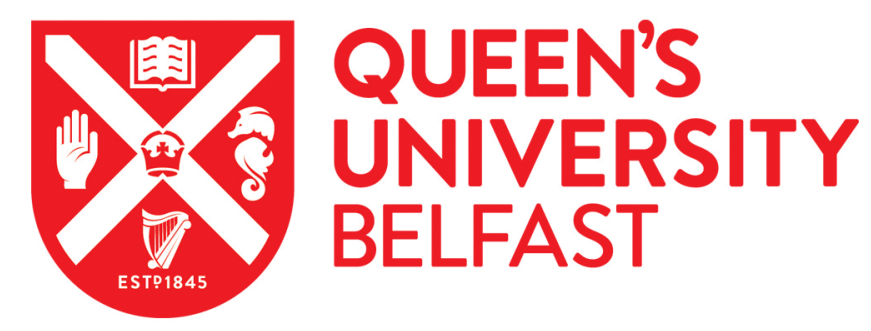

\title{
L-weakly and M-weakly compact operators and the centre
}

Bayram, E., \& Wickstead, A. W. (2017). L-weakly and M-weakly compact operators and the centre. Archiv der Mathematik, 1-9. https://doi.org/10.1007/s00013-017-1077-5

Published in:

Archiv der Mathematik

Document Version:

Publisher's PDF, also known as Version of record

Queen's University Belfast - Research Portal:

Link to publication record in Queen's University Belfast Research Portal

\section{Publisher rights}

Copyright 2017 the authors.

This is an open access article published under a Creative Commons Attribution License (https://creativecommons.org/licenses/by/4.0/), which permits unrestricted use, distribution and reproduction in any medium, provided the author and source are cited.

\section{General rights}

Copyright for the publications made accessible via the Queen's University Belfast Research Portal is retained by the author(s) and / or other copyright owners and it is a condition of accessing these publications that users recognise and abide by the legal requirements associated with these rights.

Take down policy

The Research Portal is Queen's institutional repository that provides access to Queen's research output. Every effort has been made to ensure that content in the Research Portal does not infringe any person's rights, or applicable UK laws. If you discover content in the Research Portal that you believe breaches copyright or violates any law, please contact openaccess@qub.ac.uk. 


\title{
L-weakly and M-weakly compact operators and the centre
}

\author{
E. BAYRAM AND A. W. Wickstead
}

\begin{abstract}
We extend known results concerning the centre of spaces of regular (resp. weakly compact or compact) operators between two Banach lattices to the setting of L-weakly compact and M-weakly compact operators. We also show that the L-weakly compact, M-weakly compact, and compact operators lying in the centre of a Banach lattice coincide.
\end{abstract}

Mathematics Subject Classification. 46B42, 47B60, 47B65.

Keywords. L-weakly compact operator, M-weakly compact operator, Banach lattice, Centre of ordered vector space.

1. Introduction. An operator $T$ on an ordered vector space $E$, with positive cone $E_{+}$, is called central if it is bounded by a multiple of the identity operator, i.e., there exists some scalar $\alpha>0$ such that $-\alpha x \leq T x \leq \alpha x$ holds for all $x \in E_{+}$, i.e., $-\alpha I_{E} \leq T \leq \alpha I_{E}$, where $I_{E}$ denotes the identity operator on $E$. The collection of all central operators on $E$ is called the centre of $E$ and is denoted by $Z(E)$. If $E$ is Archimedean, then $Z(E)$ is a commutative algebra and lattice with an order unit under composition, and is in fact isometrically algebra isomorphic to a dense subalgebra of some space, $C(X)$, of continuous real-valued functions on some compact Hausdorff space $X$ under pointwise multiplication and the supremum norm. Amongst many works on the centre, especially in the context of vector lattices, there have been attempts to identify the centre of various spaces of regular operators. In [9], isometric results were given by Wickstead showing that if $E$ and $F$ are Banach lattices, then there is an embedding of the algebraic tensor product $Z(E) \odot Z(F)$ into $Z\left(\mathcal{L}^{r}(E, F)\right)$ which is an isometry when $Z(E) \odot Z(F)$ is given the injective tensor product norm. That paper also gives some density results regarding this, and similar,

Author E. Bayram was supported by The Scientific and Technological Research Council of Turkey (TUBITAK) in the context of 2219-Post Doctoral Fellowship Program and by the Research Foundation of Namik Kemal University (Project No.NKUBAP.01.GA.17.108). 
embeddings. Also, in [9], related results are obtained for spaces of compact and weakly compact operators. In a slightly different vein, in [7], it is shown that compact central operators on a Banach lattice $E$ take values in the closed linear span of the atoms.

There is by now a substantial literature concerning L-weakly compact and M-weakly compact operators, especially between two Banach lattices. In this paper we modify the quoted results from $[7,9]$ to the setting of L-weakly compact and M-weakly compact operators.

2. Preliminaries. Our terminology and notations are standard, and we refer to [1] (or [2]) and [6] for unexplained definitions and properties about Banach lattices and operators on them. Recall that a non-empty bounded subset $A$ of a Banach lattice $E$ is termed $L$-weakly compact if for every disjoint sequence $\left(x_{n}\right)$ in the solid hull of $A$, we have $\left\|x_{n}\right\| \rightarrow 0$, and that an operator $T$ from a Banach space $X$ into $E$ is L-weakly compact if the image under $T$ of the closed unit ball in $X$ is L-weakly compact in $E$. An operator $T: E \rightarrow X$ is $M$ weakly compact if for every disjoint norm bounded sequence $\left(x_{n}\right)$ in $E$, we have $\left\|T x_{n}\right\| \rightarrow 0$. Throughout this paper M-weakly and L-weakly compact operators defined from $E$ into $F$ will be denoted by $\mathcal{W}_{M}(E, F)$ and $\mathcal{W}_{L}(E, F)$, respectively, whilst the spaces generated by the positive M-weakly and L-weakly compact operators will be denoted by $\mathcal{W}_{M}^{r}(E, F)$ and $\mathcal{W}_{L}^{r}(E, F)$, respectively. For conciseness, $\mathcal{W}_{L, M}(E, F)$ will represent either of the classes of L-weakly or of M-weakly compact operators, with a similar convention for $\mathcal{W}_{L, M}^{r}(E, F)$.

Under the regular norm, $\mathcal{W}_{L, M}(E, F)$ is a closed subspace of $\mathcal{L}^{r}(E, F)$. Although in general $\mathcal{W}_{M}^{r}(E, F)$ is not a vector lattice, if $F$ is Dedekind complete, then $\mathcal{W}_{M}^{r}(E, F)$ is a Dedekind complete Banach lattice under the regular norm, whilst $\mathcal{W}_{L}^{r}(E, F)$ is always a Dedekind complete Banach lattice under the regular norm.

Recall that a typical element $\phi$ of the algebraic tensor product of two Banach spaces $X$ and $Y, X \odot Y$, can be written, though not uniquely, in the form $\phi=\sum_{k=1}^{n} x_{k} \otimes y_{k}$, and then the injective norm on $X \odot Y$ is defined by

$\left\|\sum_{k=1}^{n} x_{k} \otimes y_{k}\right\|_{\lambda}=\sup \left\{\left|\sum_{k=1}^{n} f\left(x_{k}\right) \otimes g\left(y_{k}\right)\right|: f \in X^{*}, g \in Y^{*},\|f\|,\|g\| \leq 1\right\}$.

We denote $X \odot Y$ under this norm by $X \odot_{\lambda} Y$ and its completion by $X \otimes_{\lambda} Y$.

If $E$ is an Archimedean ordered vector space, then the order unit norm on $Z(E)$ is defined by

$$
\|T\|_{o}=\inf \left\{\alpha \in \mathbb{R}:-\alpha I_{E} \leq T \leq \alpha I_{E}\right\} .
$$

In this paper we will follow [9], by denoting elements of $Z(E) \odot Z(F)$ by small greek letters whilst the corresponding large letter will denote an operator on $\mathcal{L}^{r}(E, F)$ defined as follows. If $\phi=\sum_{k=1}^{n} U_{k} \otimes V_{k}$, with each $U_{k} \in Z(E)$ and $V_{k} \in Z(F)$, then we define $\Phi$ on $\mathcal{L}^{r}(E, F)$ by

$$
\Phi(T)=\sum_{k=1}^{n} V_{k} \circ T \circ U_{k}
$$


We make use of [9, Theorem 2.1] several times, so we repeat the statement here for the convenience of the reader.

Theorem 2.1. Let $E$ and $F$ be uniformly complete vector lattices, and suppose that the order dual of $E$ separates the points of $E$. If $\phi \in Z(E) \odot Z(F)$, then the corresponding operator $\Phi$ lies in $Z\left(\mathcal{L}^{r}(E, F)\right)$. Furthermore $\|\Phi\|_{o}=\|\phi\|_{\lambda}$, and the embedding may thus be extended to an isometry of $Z(E) \otimes_{\lambda} Z(F)$ into $Z\left(\mathcal{L}^{r}(E, F)\right)$ which is also an algebra and order isomorphism.

An important part of the proofs in Sects. $\S 3$ and $\S 4$ is the following observation. Because both the L-weakly compact and the M-weakly compact operators have the domination property, if $U \in Z(E), V \in Z(F)$, and $T \in \mathcal{W}_{L, M}^{r}(E, F)$, then $V T U \in \mathcal{W}_{L, M}^{r}(E, F)$. It suffices to prove this for $T \in \mathcal{W}_{L, M}(E, F)_{+}$. If $x \in E_{+}$, then

$$
|V T U x| \leq\|V\|_{o}|T U x| \leq\|V\|_{o} T\left(\|U\|_{o} x\right)=\|V\|_{o}\|U\|_{o} T x
$$

so that

$$
-\|V\|_{o}\|U\|_{o} T \leq V T U \leq\|V\|_{o}\|U\|_{o} T .
$$

It follows that $V T U \in Z\left(\mathcal{W}_{L, M}^{r}(E, F)\right)$. Thus we may define a mapping

$$
Z(E) \odot Z(F) \ni \phi \mapsto \Phi \in Z\left(\mathcal{W}_{L, M}^{r}(E, F)\right)
$$

saying that if $\phi=\sum_{k=1}^{n} U_{k} \otimes V_{k}$, with each $U_{k} \in Z(E)$ and $V_{k} \in Z(F)$, then $\Phi(T)=\sum_{k=1}^{n} V_{k} T U_{k}$ for $T \in \mathcal{W}_{L, M}^{r}(E, F)$.

Another important tool that we use is the following result of Buskes, Dodds, de Pagter, and Schep, which we again repeat for the convenience of the reader. Recall that if $E$ is a vector lattice and $A \subset E$, then $\mathcal{J}(A)$ (resp. $\mathcal{D}(A))$ is the collection of all suprema (resp. infima) in $E$ of increasing (resp. decreasing) families from $A$.

Theorem 2.2. ([4, Theorem 2.6]) If $E$ is a Banach lattice which is either Dedekind $\sigma$-complete or has a topological order unit and $F$ is a Dedekind complete Riesz space such that the order continuous linear functionals on $F$ separate the points of $F$, then

$$
\begin{aligned}
& \left\{W \in Z\left(\mathcal{L}^{r}(E, F)\right): 0 \leq W \leq I_{\mathcal{L}^{r}(E, F)}\right\} \\
& \quad=\operatorname{DJD} \mathcal{D}\left\{\Phi: \phi \in Z(E) \otimes Z(F) \text { and } 0 \leq \phi \leq I_{E} \otimes I_{F}\right\} .
\end{aligned}
$$

3. The centre of L-weakly compact operators. In [9], it is proven that the embeddings

$$
Z(E) \odot Z(F) \ni \phi \mapsto \Phi \in Z\left(\mathcal{L}^{r}(E, F)\right)
$$

and

$$
Z(E) \odot Z(F) \ni \phi \mapsto \Phi \in Z\left(\mathcal{W}^{r}(E, F)\right)
$$

are isometries where the norms on $Z\left(\mathcal{L}^{r}(E, F)\right)$ and on $Z\left(\mathcal{W}^{r}(E, F)\right)$ are order unit norms and the norm on $Z(E) \odot Z(F)$ is the injective norm. However, the embedding 


$$
Z(E) \odot Z(F) \ni \phi \mapsto \Phi \in Z\left(\mathcal{W}_{L}^{r}(E, F)\right)
$$

may not be an isometry. Recall that an L-weakly compact operator $T: E \rightarrow F$ takes values in the order continuous part, $F^{a}$, of $F . F^{a}$ is the largest ideal in $F$ on which the norm is order continuous and is closed in $F$. So if $E \neq\{0\}$ is any Banach lattice and $F=C([0,1]) \oplus c_{0}$, so that $F^{a}=\{0\} \oplus c_{0}$. Define the operator $V: F \rightarrow F$ by $V(f, x)=(f, 0)$, for $f \in C([0,1])$ and $x \in c_{0}$. Then $V \in Z(F)$ and $\left.V\right|_{F^{a}}=0$ holds so that if $\phi=I_{E} \otimes V \in Z(E) \odot Z(F)$, then the corresponding operator $\Phi: T \mapsto V \circ T \circ I_{E}=0$, so that $\|\Phi\|_{o}=0$ whilst $\|\phi\|_{\lambda} \neq 0$.

However, a smaller space is suitable for obtaining an isometric embedding.

Theorem 3.1. Let $E$ and $F$ be arbitrary Banach lattices. If $\phi \in Z(E) \odot Z\left(F^{a}\right)$, then the corresponding operator $\Phi \in Z\left(\mathcal{W}_{L}^{r}(E, F)\right)$. Furthermore, $\|\Phi\|_{o}=$ $\|\phi\|_{\lambda}$ and the embedding may be extended to an isometry of $Z(E) \otimes_{\lambda} Z\left(F^{a}\right)$ into $Z\left(\mathcal{W}_{L}^{r}(E, F)\right)$ which is also an algebra and order isomorphism.

Proof. A simple modification of the argument at the end of the previous section shows that $\Phi \in Z\left(\mathcal{W}_{L}^{r}(E, F)\right)$. According to Theorem 2.1, we only need to show that $\|\Phi\|_{o} \geq\|\phi\|_{\lambda}$. For this, it is enough to take into account that in this setting the operator $T$ constructed in the proof of Theorem 2.1, given in [9, Theorem 2.1], is $L$-weakly compact as $y \in F^{a}$ because we have $Q \in Z\left(F^{a}\right)$.

However, this embedding in the previous theorem is not suitable for obtaining density results. Let $E$ be an infinite-dimensional Banach lattice with trivial centre and $F=\mathbb{R}$. Then, $Z(E) \odot Z\left(F^{a}\right)$ is one-dimensional whilst $Z\left(\mathcal{W}_{L}^{r}(E, F)\right)=Z\left(E^{*}\right)$ will be infinite-dimensional.

For this reason, to obtain density results the isometric result should be reformulated. Before presenting the density results, we will give two more isometric embedding results. However, we should change the definition of the map $\phi \mapsto \Phi$. Let us take

$$
\phi=\sum_{k=1}^{n} U_{k} \otimes V_{k} \in Z\left(E^{*}\right) \odot Z\left(F^{a}\right),
$$

and define $\Phi$ on $\mathcal{W}_{L}^{r}(E, F)$ by

$$
\Phi(T)=\left(\left.\sum_{k=1}^{n} V_{k} T^{* *} U_{k}^{*}\right|_{E}\right) .
$$

Theorem 3.2. Let $E$ and $F$ be arbitrary Banach lattices. If $\phi \in Z\left(E^{*}\right) \odot$ $Z\left(F^{a}\right)$, then the corresponding operator $\Phi \in Z\left(\mathcal{W}_{L}^{r}(E, F)\right)$. Furthermore, $\|\Phi\|_{o}=\|\phi\|_{\lambda}$ and the embedding may be extended to an isometry of $Z\left(E^{*}\right) \otimes_{\lambda}$ $Z\left(F^{a}\right)$ into $Z\left(\mathcal{W}_{L}^{r}(E, F)\right)$ which is also an algebra and order isomorphism.

Proof. It is enough to show that $\|\Phi\|_{o} \geq\|\phi\|_{\lambda}$ by Theorem 2.1. This can be seen easily from [9, Corollary 3.4], because the operator $T$ constructed in the proof is a $L$-weakly compact operator as $y \in F^{a}$ because $Q \in Z\left(F^{a}\right)$. 
For the density result, an embedding $Z\left(E^{* *}\right) \odot Z\left(F^{a}\right)$ into $Z\left(\mathcal{W}_{L}^{r}(E, F)\right)$ is more appropriate, at least when combined with the assumption of order continuity condition of $E^{*}$. However, we again need to reformulate the definition of the map $\phi \mapsto \Phi$. Let us take

$$
\phi=\sum_{k=1}^{n} U_{k} \otimes V_{k} \in Z\left(E^{* *}\right) \odot Z\left(F^{a}\right),
$$

and define $\Phi$ on $\mathcal{W}_{L}^{r}(E, F)$ by

$$
\Phi(T)=\left(\left.\sum_{k=1}^{n} V_{k} T^{* *} U_{k}\right|_{E}\right) .
$$

Corollary 3.3. Let $E$ and $F$ be arbitrary Banach lattices with $E^{*}$ having an order continuous norm. If $\phi \in Z\left(E^{* *}\right) \odot Z\left(F^{a}\right)$, then the corresponding operator $\Phi \in Z\left(\mathcal{W}_{L}^{r}(E, F)\right)$. Furthermore, $\|\Phi\|_{o}=\|\phi\|_{\lambda}$ and the embedding may be extended to an isometry of $Z(E) \otimes_{\lambda} Z\left(F^{a}\right)$ into $Z\left(\mathcal{W}_{L}^{r}(E, F)\right)$ which is also an algebra and order isomorphism.

Proof. If $E^{*}$ has order continuous norm, then $\left\{T^{*}: T \in Z\left(E^{*}\right)\right\}=Z\left(E^{* *}\right)$, so the proof follows immediately from the previous result.

Theorem 3.4. Let $E$ and $F$ be Banach lattices with the dual space $E^{*}$ of $E$ having an order continuous norm. Then

$$
\begin{aligned}
& \left\{W \in Z\left(\mathcal{W}_{L}^{r}(E, F)\right): 0 \leq W \leq I_{\mathcal{W}_{L}^{r}(E, F)}\right\} \\
& \quad=\operatorname{DJDJJ}\left\{\Phi: \phi \in Z\left(E^{* *}\right) \odot Z\left(F^{a}\right) \text { and } 0 \leq \phi \leq I_{E^{* *}} \otimes I_{F^{a}}\right\},
\end{aligned}
$$

and hence $\left\{\Phi: \phi \in Z\left(E^{* *}\right) \odot Z\left(F^{a}\right)\right\}$ is dense in $Z\left(\mathcal{W}_{L}^{r}(E, F)\right)$ for the strong operator topology.

Proof. In this setting, we know that $\mathcal{W}_{L}^{r}(E, F)$ and $\left\{T^{*}: T \in \mathcal{W}_{L}^{r}(E, F)\right\}$ are ideals in $\mathcal{L}^{r}(E, F)$ and $\mathcal{L}^{r}\left(F^{*}, E^{*}\right)$, respectively. Let $W \in Z\left(\left\{T^{*}: T \in \mathcal{W}_{L}^{r}(E, F)\right\}\right)$. By [8, Theorem 1], there is an extension $W^{\prime} \in$ $Z\left(\mathcal{L}^{r}\left(\left(F^{a}\right)^{*}, E^{*}\right)\right)$. Since $\left(F^{a}\right)^{*}$ and $E^{*}$ are Dedekind complete Banach lattices, by Theorem 2.2, there is an approximation for $Z\left(\mathcal{L}^{r}\left(\left(F^{a}\right)^{*}, E^{*}\right)\right)$ by $\left\{\Phi: \phi \in Z\left(\left(F^{a}\right)^{*}\right) \odot Z\left(E^{*}\right)\right\}$, and hence for $Z\left(\left\{T^{*}: T \in \mathcal{W}_{L}^{r}(E, F)\right\}\right)$. Indeed, by Theorem 3.1,

$$
W^{\prime} \in \mathcal{D J D} \mathcal{D} \mathcal{J}\left\{\Phi: \phi \in Z\left(\left(F^{a}\right)^{*}\right) \odot Z\left(E^{*}\right) \text { and } 0 \leq \phi \leq I_{\left(F^{a}\right)^{*}} \otimes I_{E^{*}}\right\},
$$

and it follows that

$$
\begin{aligned}
W= & \left.W^{\prime}\right|_{\left\{T^{*}: T \in \mathcal{W}_{L}^{r}(E, F)\right\}} \\
& \in \mathcal{D J D J}\left\{\left.\Phi\right|_{\left\{T^{*}: T \in \mathcal{W}_{L}^{r}(E, F)\right\}}: \phi \in Z\left(\left(F^{a}\right)^{*}\right) \odot Z\left(E^{*}\right)\right. \text { and } \\
& \left.0 \leq \phi \leq I_{\left(F^{a}\right)^{*}} \otimes I_{E^{*}}\right\} .
\end{aligned}
$$

Let $\phi=\sum_{k=1}^{n} V_{k} \otimes U_{k} \in Z\left(\left(F^{a}\right)^{*}\right) \odot Z\left(E^{*}\right)$. As $F^{a}$ has order continuous norm $V_{k} \in Z\left(\left(F^{a}\right)^{*}\right)={ }^{*} Z\left(F^{a}\right)$. Since $E^{*}$ has order continuous norm, $E^{*}$ is an ideal in $E^{* * *}$. If $W_{k}=U_{k}^{*}$, then $W_{k} \in Z\left(E^{* *}\right)$, that is, $W_{k}^{*}=U_{k}^{* *} \in$ $Z\left(E^{* * *}\right)$. It follows that $\left.W_{k}^{*}\right|_{E^{*}}=\left.U_{k}^{* *}\right|_{E^{*}}=U_{k} \in Z\left(E^{*}\right)$, so $\phi=\sum_{k=1}^{n} V_{k} \otimes$ 
$\left.W_{k}^{*}\right|_{E^{*}} \in Z\left(\left(F^{a}\right)^{*}\right) \odot Z\left(E^{*}\right)$. This gives the desired order approximation for $Z\left(\mathcal{W}_{L}^{r}(E, F)\right)$. Approximation for the strong operator topology can be seen by the fact that the regular norm on $\mathcal{W}_{L}^{r}(E, F)$ is order continuous as $E^{*}$ has order continuous norm ([3, Theorem 3.1]).

4. The center of $M$-weakly compact operators. Similar to the $L$-weakly compact operators case, the embedding

$$
Z(E) \odot Z(F) \ni \phi \mapsto \Phi \in Z\left(\mathcal{W}_{M}^{r}(E, F)\right)
$$

may not be an isometry where the norm on $Z\left(\mathcal{W}_{M}^{r}(E, F)\right)$ is order unit norm and the norm on $Z(E) \odot Z(F)$ is injective norm. To see this, let us consider an arbitrary Banach lattice $F \neq\{0\}, E=L^{1}([0,1]) \oplus \ell_{1}$, and the operator $U: E \rightarrow E$ defined by $U(f, x)=(f, 0)$, where $f \in L^{1}([0,1])$ and $x \in \ell_{1}$. Clearly, $0 \neq U \in Z(E)$ whilst $U^{*}$ is the zero operator on $\left(E^{*}\right)^{a}$. Thus, for the non-zero tensor $\phi=U \otimes I_{F} \in Z(E) \odot Z(F)$, the corresponding operator $\Phi: T \mapsto I_{F} \circ T \circ U=T \circ U$. For every $M$-weakly compact operator $T, T^{*}$ is L-weakly compact (by [6, Proposition 3.6.11]) so that $T^{*}:\left(F^{*}\right) \subseteq\left(E^{*}\right)^{a}$, and hence $\Phi(T)^{*}=U^{*} \circ T^{*}=0$. Hence $\Phi(T)=0$, and thus $\Phi=0$ even though $\phi \neq 0$.

If

$$
\phi=\sum_{k=1}^{n} U_{k} \otimes V_{k} \in Z\left(\left(E^{*}\right)^{a}\right) \odot Z(F),
$$

define $\Phi$ on $\mathcal{W}_{M}^{r}(E, F)$ by

$$
\Phi(T)=\left(\left.\sum_{k=1}^{n} V_{k} T^{* *} U_{k}^{*}\right|_{E}\right) .
$$

Theorem 4.1. Let $E$ and $F$ be arbitrary Banach lattices. If $\phi \in Z\left(\left(E^{*}\right)^{a}\right) \odot$ $Z(F)$, then the corresponding operator $\Phi \in Z\left(\mathcal{W}_{M}^{r}(E, F)\right)$. Furthermore, $\|\Phi\|_{o}=\|\phi\|_{\lambda}$ and the embedding may be extended to an isometry of $Z\left(\left(E^{*}\right)^{a}\right) \otimes_{\lambda}$ $Z(F)$ into $Z\left(\mathcal{W}_{M}^{r}(E, F)\right)$ which is also an algebra and order isomorphism.

Proof. It is enough to show that $\|\Phi\|_{o} \geq\|\phi\|_{\lambda}$ by Theorem 2.1. This can be seen easily from [9, Corollary 3.4], because the operator $T$ constructed in the proof of $\left[9\right.$, Theorem 2.1] is $M$-weakly compact as $f \in\left(E^{*}\right)_{+}^{a}$ because $P \in Z\left(\left(E^{*}\right)^{a}\right)$.

In order to obtain a density result here, recall that ${ }^{*} Z\left(E^{*}\right)$ is simply $\left\{T^{*}\right.$ : $\left.T \in Z\left(E^{*}\right)\right\}$, which is certainly a subset (in general proper) of $Z\left(E^{* *}\right)$. The next two results use the embedding

$$
{ }^{*} Z\left(\left(E^{*}\right)^{a}\right) \odot Z(F) \hookrightarrow Z\left(\mathcal{W}_{M}^{r}(E, F)\right)
$$

mapping

$$
\phi=\sum_{k=1}^{n} U_{k} \otimes V_{k} \in^{*} Z\left(\left(E^{*}\right)^{a}\right) \odot Z(F)
$$


to an operator $\Phi$ defined on $\mathcal{W}_{M}^{r}(E, F)$ by

$$
\Phi(T)=\left(\left.\sum_{k=1}^{n} V_{k} T^{* *} U_{k}\right|_{E}\right) .
$$

The following result follows directly from Theorem 4.1.

Corollary 4.2. Let $E$ and $F$ be arbitrary Banach lattices. If $\phi \in{ }^{*} Z\left(\left(E^{*}\right)^{a}\right) \odot$ $Z(F)$, then the corresponding operator $\Phi \in Z\left(\mathcal{W}_{M}^{r}(E, F)\right)$. Furthermore, $\|\Phi\|_{o}=\|\phi\|_{\lambda}$ and the embedding may be extended to an isometry of ${ }^{*} Z\left(\left(E^{*}\right)^{a}\right) \otimes_{\lambda} Z(F)$ into $Z\left(\mathcal{W}_{M}^{r}(E, F)\right)$ which is also an algebra and order isomorphism.

Theorem 4.3. Let $E$ and $F$ be Banach lattices with $F$ having an order continuous norm. Then

$$
\begin{aligned}
& \left\{W \in Z\left(\mathcal{W}_{M}^{r}(E, F)\right): 0 \leq W \leq I_{\mathcal{W}_{M}^{r}(E, F)}\right\} \\
& \quad=\operatorname{DJDJJ}\left\{\Phi: \phi \in{ }^{*} Z\left(\left(E^{*}\right)^{a}\right) \odot Z(F) \text { and } 0 \leq \phi \leq I_{\left(E^{*}\right)^{a}}^{*} \otimes I_{F}\right\}
\end{aligned}
$$

and hence $\left\{\Phi: \phi \in{ }^{*} Z\left(\left(E^{*}\right)^{a}\right) \odot Z(F)\right\}$ is dense in $Z\left(\mathcal{W}_{M}^{r}(E, F)\right)$ for the strong operator topology.

Proof. It is known that in this setting, $\mathcal{W}_{M}^{r}(E, F)$ and $\left\{T^{*}: T \in \mathcal{W}_{M}^{r}(E, F)\right\}$ are ideals in $\mathcal{L}^{r}(E, F)$ and $\mathcal{L}^{r}\left(F^{*},\left(E^{*}\right)^{a}\right)$, respectively. Let $W \in Z\left(\left\{T^{*}: T \in \mathcal{W}_{M}^{r}(E, F)\right\}\right)$. By [8, Theorem 1], there is a extension $W^{\prime} \in Z\left(\mathcal{L}^{r}\left(F^{*},\left(E^{*}\right)^{a}\right)\right)$. Since $F^{*}$ and $\left(E^{*}\right)^{a}$ are Dedekind complete Banach lattices, by [9, Theorem 3.1], there is an approximation for $Z\left(\mathcal{L}^{r}\left(F^{*},\left(E^{*}\right)^{a}\right)\right)$ by $\left\{\Phi: \phi \in Z\left(F^{*}\right) \odot Z\left(\left(E^{*}\right)^{a}\right)\right\}$, and hence for $Z\left(\left\{T^{*}: T \in \mathcal{W}_{M}^{r}(E, F)\right\}\right)$. Indeed, by $[9$, Theorem 3.1],

$$
W^{\prime} \in \mathcal{D J D J}\left\{\Phi: \phi \in Z\left(F^{*}\right) \odot Z\left(\left(E^{*}\right)^{a}\right) \text { and } 0 \leq \phi \leq I_{F^{*}} \otimes I_{\left(E^{*}\right)^{a}}\right\},
$$

and it follows that

$$
\begin{aligned}
W= & \left.W^{\prime}\right|_{\left\{T^{*}: T \in \mathcal{W}_{M}^{r}(E, F)\right\}} \\
& \in \mathcal{D J D J J}\left\{\left.\Phi\right|_{\left\{T^{*}: T \in \mathcal{W}_{M}^{r}(E, F)\right\}}: \phi \in Z\left(F^{*}\right) \odot Z\left(\left(E^{*}\right)^{a}\right)\right. \text { and } \\
& \left.0 \leq \phi \leq I_{F^{*}} \otimes I_{\left(E^{*}\right)^{a}}\right\} .
\end{aligned}
$$

Let $\phi=\sum_{k=1}^{n} V_{k} \otimes U_{k} \in Z\left(F^{*}\right) \odot Z\left(\left(E^{*}\right)^{a}\right)$. As $F$ has order continuous norm $V_{k} \in Z\left(F^{*}\right)={ }^{*} Z(F)$. Let us take $W_{k}=U_{k}^{*}$. Then $W_{k} \in{ }^{*} Z\left(\left(E^{*}\right)^{a}\right)$,

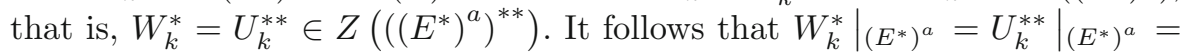
$U_{k} \in Z\left(\left(E^{*}\right)^{a}\right)$ as $\left(E^{*}\right)^{a}$ is an ideal in $\left(\left(E^{*}\right)^{a}\right)^{* *}$, so $\phi=\left.\sum_{k=1}^{n} V_{k} \otimes W_{k}^{*}\right|_{E^{*}} \in$ ${ }^{*} Z(F) \odot Z\left(\left(E^{*}\right)^{a}\right)$. This gives the desired order approximation for $Z\left(\mathcal{W}_{M}^{r}(E, F)\right)$. Approximation for the strong operator topology can be seen from the fact that the regular norm on $\mathcal{W}_{M}^{r}(E, F)$ is order continuous as $F$ has order continuous norm $([3$, Theorem 3.2]). 
5. Central L- and M-weakly compact operators. For a compact Hausdorff space $K, C^{\infty}(K)$ consist all continuous functions $f: K \rightarrow[-\infty, \infty]$ which are finite on an open dense set of $K$. The representation of Davies in [5, Theorem $10]$ states that if $E$ is an Banach lattice with a quasi-interior point $e$, then there exists a representation of $E$ as an ideal in $C^{\infty}(K)$ for a compact Hausdorff space $K$ and the quasi-interior point is represented with the function $1_{K}$ takes value constantly 1 on $K$. On the other hand, [7, Theorem 4.1] represents compact central operators and states that a central operator $T \in Z(E)$ is compact if and only if $T$ takes values in the closed linear span of the atoms of $E_{+}$. We conclude the paper by showing that the same result holds for L-weakly compact and M-weakly compact central operators.

Theorem 5.1. If $E$ is a Banach lattice and $T \in Z(E)$, then $T$ is compact if and only if $T$ is L-weakly compact if and only if $T$ is $M$-weakly compact.

Proof. Let $T \in Z(E)$ be M-weakly compact. Suppose first that the Banach lattice $E$ has a quasi-interior point $u$. Hence, there exists a representation of $E$ as an ideal in $C^{\infty}(K)$ for a compact Hausdorff space $K$ such that $u \mapsto 1_{K}$. Also, we can identify $Z(E)$ with $C(K)$. Let $\alpha>0$, and $\phi_{T} \in C(K)$ denotes the function corresponding to $T$. Define $U=\left\{k \in K: \phi_{T}(k)>\alpha\right\}$. We claim that $U$ is finite. In the contrary case, by Urysohn's lemma, there exists a disjoint sequence $\left(f_{n}\right) \subset\left[0,1_{K}\right]$ supported by $U$. Let $g_{n}=\frac{f_{n}}{\left\|f_{n}\right\|}$, so $\left(g_{n}\right)$ is a disjoint sequence in the closed unit ball $B_{E}$. Thus, $T g_{n}=\phi_{T} g_{n} \geq \alpha g_{n}$, so $\left\|T g_{n}\right\| \geq \alpha$. This contradicts the $M$-weak compactness of $T$. It follows that $U$ is finite. Note also that a Banach lattice with a quasi-interior point has at most countably many atoms. Thus, analogously to [7, Theorem 4.1], there exist atoms $e_{n} \in E$ and $\lambda_{n} \in \mathbb{R}$ with $\lambda_{n} \rightarrow 0$ and

$$
T x=\sum_{n=1}^{\infty} \lambda_{n} P_{n}(x)
$$

where $x \in X$ and $P_{n}: X \rightarrow \mathbb{R} e_{n}$ is band projection. It follows that $T$ is compact.

Suppose that $E$ has no quasi-interior point. Let $J$ be the closed ideal generated by $0 \neq x \in E_{+}$. Clearly, $\left.T\right|_{J} \in Z(J)$ and from the first part of proof, as $x$ is a quasi interior point of $J, T x$ belongs to the closed linear span generated by atoms of $J$, and therefore of $E$. If the atoms are $\left\{e_{a}: a \in \Lambda\right\}$, then there exists $\lambda_{a} \in \mathbb{R}$ with $T e_{a}=\lambda_{a} e_{a}$. We claim that $\Gamma=\left\{a \in A: \lambda_{a} \geq \alpha>0\right\}$ is finite. If not, let $a_{n} \in \Gamma$, then $u=\sum_{n=1}^{\infty} \frac{e_{a_{n}}}{2^{n}} \in E$. Hence, the ideal generated by $u$ in $E$ has quasi-interior point, so $T e_{a_{n}} \geq \alpha e_{a_{n}}$. This contradicts $T$ being $M$-weakly compact. Hence, we may repeat the first part of proof to see that $T$ is compact.

If $T \in Z(E)$ is L-weakly compact, then $T^{*}$ is an M-weakly compact central operator, so is compact, and hence $T$ is compact.

Finally, a compact central operator $T$ takes values in the closed linear span of the atoms from which it follows easily that it is L-weakly compact. Also, $T^{*}$ being compact and central implies that $T^{*}$ is L-weakly compact, and therefore $T$ is M-weakly compact. 
Open Access. This article is distributed under the terms of the Creative Commons Attribution 4.0 International License (http://creativecommons.org/licenses/ by/4.0/), which permits unrestricted use, distribution, and reproduction in any medium, provided you give appropriate credit to the original author(s) and the source, provide a link to the Creative Commons license, and indicate if changes were made.

\section{References}

[1] C. D. Aliprantis and O. Burkinshaw, Positive Operators, Pure and Applied Mathematics, 119, Academic Press, Inc., Orlando, FL, 1985.

[2] C. D. Aliprantis and O. Burkinshaw, Positive Operators, Springer, Dordrecht, 2006.

[3] E. Bayram And A. W. Wickstead, Banach lattices of L-weakly and M-weakly compact operators, Arch. Math. (Basel) 108 (2017), 293-299.

[4] G. J. H. M. Buskes, P. G. Dodds, B. de Pagter, And A. R. Schep, Up-down theorems in the centre of $\mathcal{L}_{\mathrm{b}}(\mathrm{E}, \mathrm{F})$, Nederl. Akad. Wetensch. Indag. Math. 48 (1986), 1-9.

[5] E. B. DAviEs, The Choquet theory and representation of ordered Banach spaces, Illinois J. Math. 13 (1969), 176-187.

[6] P. Meyer-Nieberg, Banach Lattices, Universitext, Springer-Verlag, Berlin, 1991.

[7] A. W. Wickstead, The ideal centre of a Banach lattice, Proc. Roy. Irish Acad. Sect. A 76 (1976), 15-23.

[8] A. W. Wickstead, Extensions of orthomorphisms, J. Austral. Math. Soc. Ser. A 29 (1980), 87-98.

[9] A. W. Wickstead, The centre of spaces of regular operators, Math. Z. 241 (2002), 165-179.

E. BAYRAM

Department of Mathematics, Faculty of Science and Arts,

Namik Kemal University,

Tekirdag,

Turkey

e-mail: ebayram@nku.edu.tr

A. W. WiCKSTEAD

Mathematical Sciences Research Centre,

Queen's University Belfast,

Belfast, BT7 1NN,

Northern Ireland, UK

e-mail: A.Wickstead@qub.ac.uk

Received: 12 April 2017 